OnLine Journal of Biological Sciences 11 (1): 7-12, 2011

ISSN 1608-4217

(C) 2011 Science Publications

\title{
Silicone Modeling of the Interior Spaces of Hollow Organs: Use in Dog and Manatee Respiratory Tract and in a Beef Heart
}

\author{
${ }^{1}$ Charles J. Grossman, ${ }^{1}$ Richard Hamilton, ${ }^{1}$ Lisa A. Close-Jacob, \\ ${ }^{2}$ Martine De Wit and ${ }^{3}$ Jeffery Werwa \\ ${ }^{1}$ Department of Biology, Xavier University, \\ Midwest Florida Manatee Research Project, \\ 3800 Victory Parkway Cincinnati, Ohio 45207, USA \\ ${ }^{2}$ Florida Fish and Wildlife Conservation Commission, \\ Fish and Wildlife Research Institute, \\ Marine Mammal Pathobiology Laboratory, \\ 3700 54th Avenue South St. Petersburg, Florida 33711, USA \\ ${ }^{3}$ Cincinnati Animal Medical Center, \\ 8350 Plainfield Road Cincinnati, Ohio 45236, USA
}

\begin{abstract}
Problem statement: The mechanism, by which the Florida manatee (Trichechus manatus latirostris) vocalizes, remains unknown because the manatee larynx does not contain true vocal cords. Since sound can be generated when air passes through a narrow respiratory structure we needed to visualize the internal anatomy of manatee respiratory tract to locate any candidate regions for study. Approach: To visualize the internal anatomy of upper and lower manatee respiratory tract we have developed a rapid but accurate method of modeling these structures using liquid silicone. We first tested this technique on the respiratory structure of a cadaver dog and then applied it to two small manatees which had died through natural causes. Incisions were made in the trachea of both dog and manatees and commercially available liquid silicone was then forced into the upper and lower respiratory tracts used a slightly modified common automobile grease gun. The animals were then refrigerated overnight and the silicone was allowed to cure for a period of $24 \mathrm{~h}$. Results: In dog, we removed cured silicone model by applying mild force to it after surgically opening the nasal cavity. In the manatees some dissection was necessary for release of mold from the upper nasal cavity, but only mild force was necessary with no dissection to release silicone model from the lower tract. Because the models created exhibited great accuracy and fine structure, including presence of tertiary bronchi in the manatee respiratory tract, we realized that the technique was applicable for use in other hollow organs. We applied this method to the visualization of internal structure of a fresh beef heart and were pleased with the accuracy and detail of model produced. Conclusion: We suggest that this technique can be adopted for three-dimensional visualization of the internal structure and volume estimation of many hollow organs in a wide variety of organisms with both minimal effort and cost.
\end{abstract}

Key words: Silicone modeling technique, Florida Manatee (Trichechus manatus latirostri), respiratory systems, upper respiratory, primary bronchi, biological organs, silicone model, modeling compound

\section{INTRODUCTION}

In our search for the mechanism by which the Florida manatee (Trichechus manatus latirostris) produces vocalizations while submerged, we have developed a silicone modeling technique to visualize the internal architecture of hollow biological organs.
We first applied this technique to the internal anatomy of the dog respiratory system and discovered that the technique worked well. We then used it to define the internal anatomy of the upper and lower portions of two small manatee respiratory systems. Although some have reported that the manatee larynx lacks true vocal cords (Murie 1872; Hill, 1945) the animals do in fact

Corresponding Author: Charles J. Grossman, Department of Biology, Xavier University, Midwest Florida Manatee Research Project, 3800 Victory Parkway Cincinnati, Ohio 45207, USA Tel: 513-745-3623 Fax: 513-745-1079 
vocalize. We hypothesized that the most likely area of narrowing where sound vibrations could be generated should be in the larynx at the glottal opening. In reviewing the three dimensional silicone models of the hollow space within the upper and lower manatee respiratory system, such a narrow region at this location was indeed observed. Our laboratory is currently involved in a series of studies to define the exact laryngeal mechanism which can generate vocalizations and we hope to be ready to publish these results in the near future. However, given the accuracy and fine detail produced in the silicone models of the manatee respiratory apparatus, we reasoned that this technique could be universally adaptable for use in other hollow biological organs. To demonstrate that this was the case we tested it on a fresh bovine heart and were gratified by the excellent and accurate three-dimensional anatomical architecture of the model.

\section{MATERIALS AND METHODS}

Silicon modeling of the interior spaces of $\mathrm{dog}$ and manatee respiratory systems: Liquid silicone in the form of MoldRite 25 (SP700501R) was purchased from Environ Molds (ArtMolds Sculpture Studio, 18 Bank Street, Summit NJ 07901, 1-866-278-6653). An amount of this silicone was mixed with the catalyst provided ( $10 \%$ catalyst by weight). The working time of this modeling compound is roughly one hour and $24 \mathrm{~h}$ is required for complete curing. Note that the curing process does not require the presence of air, which makes it suitable for modeling the largely enclosed cavities of anatomical organs. The resulting cured model is sufficiently flexible to allow for relative ease of extraction, yet robust enough to withstand repeated handling and examination.

We purchased a standard pump action, metal grease gun from an automotive supply store and removed the valve tip from the end of the metal injection tube. The spring loaded plunger was then pulled back and locked in position and the siliconeplus-catalyst mixture was loaded into the grease gun by unscrewing the barrel end. After the mixture was loaded, the plunger was released and this supplied pressure on the silicone in the body of the gun.

We first tested this procedure on the cadaver of a dog, approximately 30 inches in length and $60 \mathrm{lbs}$, which had been euthanized for unrelated reasons by a local animal shelter which was disposing of it. The dog was placed on its back with the trachea exposed and a slit was made in the upper trachea. The tip of the grease gun was inserted into the tracheal slit in a caudal direction and silicone compound was then injected by pumping the lever of the gun until the mixture began to leak around the tip. We also noted that some of the silicone began leaking out of the nares because it was able to flow in a retrograde fashion through the partially open glottis. The tip of the gun was then attached to a rubber tube which was placed first into one naris and compound was injected. This procedure was also repeated in the other naris. We noted that additional silicone again leaked out of the tracheal slit during this process, indicating that the glottis was indeed at least partially open in this animal. We then placed the body in ice for $24 \mathrm{~h}$ and after the mixture had set, we dissected the upper respiratory tract and removed the complete silicone mold. We soaked this resulting model in a dilute solution of Clorox to neutralize any residual bacteria and odor, then rinsed it in water and allowed it to air dry.

We employed the same basic technique on the two small manatee carcasses that were made available to us at the Florida Fish and Wildlife Conservation Commission, Fish and Wildlife Research Institute, Marine Mammal Pathobiology Laboratory in St. Petersburg Florida. Manatee 1 (MSW0780, necropsy date Oct 7-8, 2008) was a female, $144 \mathrm{~cm}$ long; death was from natural causes. Manatee 2 (MNW0848, necropsy date Oct 7-8, 2008) was also a female, $158 \mathrm{~cm}$ long; death was from natural causes and the body was in partial decomposition. The animals were placed on their backs, their tracheae exposed and slits were made (Fig.1-8). However, in manatees (unlike dogs), the trachea is very short below the laryngeal connection and almost immediately bifurcates into the right and left primary bronchi. We injected the silicone through the tracheal slits (Fig. 2), but noted that none of the compound leaked out of the nares of either animal because in both cases the glottis was completed closed (unlike in the case of the dog we studied earlier). In the smaller postnatal manatee carcass, we also injected silicone into both primary bronchi as well as up into the laryngeal region (Fig. 3). On the larger of the two animals we did not do this bronchial injection (results not shown). In both animals we then injected additional silicone through their nares using the metal pipe on the grease gun (Fig. 3). Here, no rubber tube was required because the size of the nares, even on these small manatees, allowed the metal pipe to be inserted directly. The animals were then placed in a walk-in refrigerator for $24 \mathrm{~h}$ to allow the silicone to set.

After 24 h, we dissected both animals (Fig. 4-8) and extracted the silicone molds. We surgically transected the larynx in both animals with a single incision and then withdrew the cured model from the larynx, trachea, primary bronchi and esophagus by gently pulling it out of these structures. 


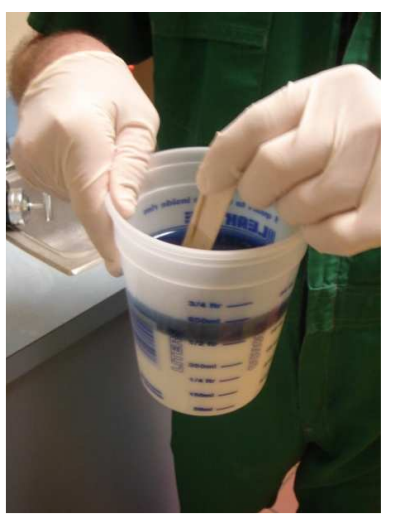

Fig. 1: Preparing the silicon modeling compound. 10\% catalyst by weight (blue layer) is being mixed with the silicon which will then be loaded into the grease gun for injection

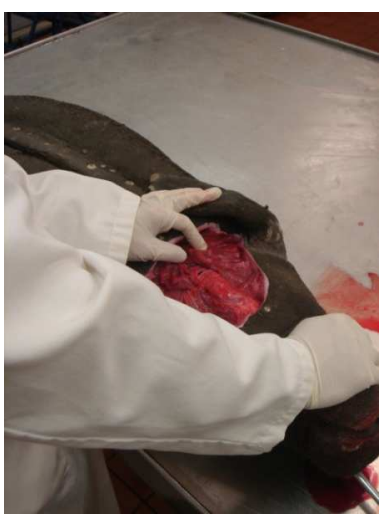

Fig. 2: Here the trachea in the smaller of the two animals is exposed and a slit cut. The slit will allow air to escape as the silicon is injected into the nares. The tip of the grease gun can be seen at the lower right inserted into the nares. The tracheal slit will then also be used to inject silicon down into the lower airway

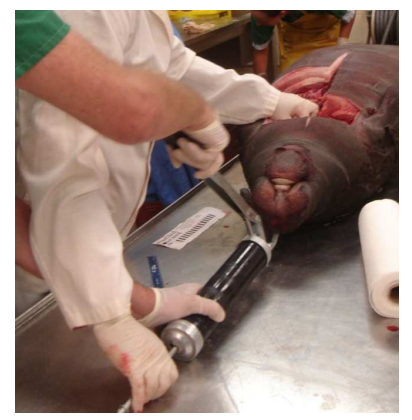

Fig. 3: View as the silicon is being injected into the nares of the smaller manatee

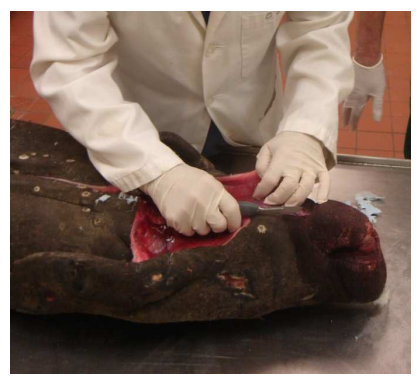

Fig. 4: After $24 \mathrm{~h}$ in the cold the trachea is fully opened in preparation for removal of the cured silicon model

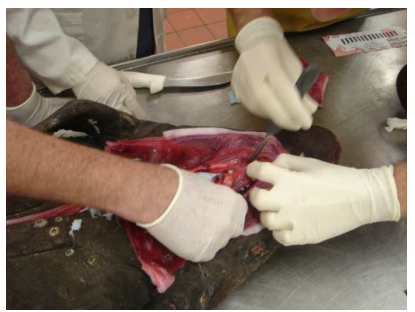

Fig. 5: Here the trachea and a portion of the upper airway are being opened. The cured silicon (blue gray in color) can be seen

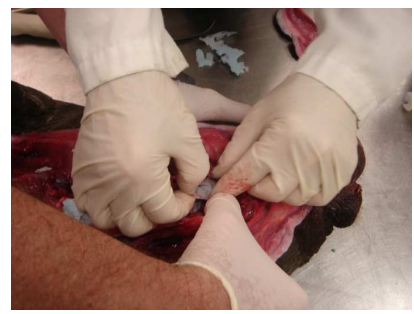

Fig. 6: The cured silicon model is carefully being extracted. Only minimal force is required for removal

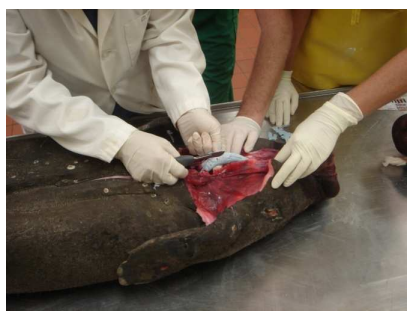

Fig. 7: The model is sliding out of the lower airway. To remove the model from the nasal area slightly more force was required but it was not necessary to employ surgery. In our earlier studies in the dog, surgery was necessary to release the model from the nasal cavity 


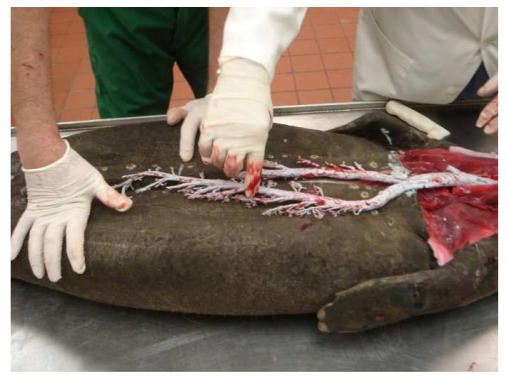

Fig. 8: The entire silicon model from the lower airway has been removed. Here the short trachea is exposed as are the two long primary bronchi and the secondary and tertiary bronchi

As with the dog, we were surprised by the incredible accuracy seen in the models. We were especially gratified by the definition of the secondary and even tertiary bronchi (Fig. 8). To remove the silicone model from the upper respiratory tract, we had to dissect out the nasal cavity on the smaller animal. However, with the larger animal (model not shown), we simply applied medium pressure on the cured silicone and the entire structure was extracted with no need for dissection (Fig. 7-9a) and no damage to the model. As in the preliminary studies in the dog, we soaked the models from these manatees in a dilute solution of Clorox and rinsed them in water.

Silicon modeling of the interior spaces of a bovine heart: To demonstrate that this silicone modeling technique is easily adaptable for use with other hollow organs, we applied it to model the internal spaces of a beef heart. A fresh specimen was obtained from a local slaughter house. We cut two small slits in the left and right ventricles near the apex of the heart to allow air to be vented as modeling compound was introduced. Silicone was pumped into the heart by placing the tip of the grease gun into the attached vessels and slowly forcing the material into the left and right atria and ventricles. However, because some of the valves were closed, silicone was also introduced into the ventricles by placing the tip of the grease gun into the slits we had made near the apex as described above. The steady pressure applied as the compound was loaded into the ventricles also forced silicone into the attached coronary arteries, as can be visualized in the model that was produced (Fig. 10a-c). After allowing the silicon to cure for $24 \mathrm{~h}$, the completed mold was removed by a combination of dissection of the muscle tissue and extraction of the cured material by applying mild tension. The silicone model was then immersed in Clorox, washed in water and dried.

\section{RESULTS AND DISCUSSION}

Interior spaces of the dog and manatee respiratory systems: In Fig. 9a the silicone model from the smaller of the two manatees (MSW0780) is shown, as are the results from the dog (Fig. 9b). Here the shape of the interior spaces of the nasal cavity, oral cavity, pharynx, larynx and trachea are all apparent. Furthermore, the location of the glottis in the manatee (Fig. 9a expanded) and dog (Fig. 9b expanded) can be seen as the narrow region between the pharynx and trachea. Of particular interest is the presence of the very large Eustachian tubes that are clearly visible projecting from the pharyngeal region in the manatee model (Fig. 9a and 9a expanded).

Our technique also demonstrates that the nasal cavity in the dog possesses highly complex turbinate (Fig. 9b), which are lacking in the upper respiratory tract of the manatee (Fig. 9a). Additionally, the location of a nasal septum in both manatee (Fig. 9a) and dog (Fig. 9b) can be inferred from the separation between the left and right nasal cavities in both models.

Interior spaces of a beef heart: Given the ease with which this technique can be applied to the hollow respiratory structures, we further employed it to delineate the three-dimensional spaces within a fresh beef heart. In Fig. 10a the right atria and right ventricular spaces are modeled along with a portion of the right coronary artery and a portion of the Left Anterior Descending (LAD) coronary artery (right lateral view), while in Fig. 10b (ventral view) portions of both the right and left ventricles, as well as the separation between them produced by the presence of the septum, can be visualized. In Fig. 10c (dorsal view) the left atria and ventricular spaces are seen along with the LAD coronary artery and some of its branches. In Fig. 10a-c, the aorta can be seen, along with one of its major branches (Fig. 10a and b). Also, although it is not shown clearly in Fig. 10c, it is possible to deflect the pliable silicone material away from the modeled left ventricle to visualize the location of the aortic semilunar valve. The location of the pulmonary artery can also be seen in Fig. 10b as it exits the right ventricle and the location of the pulmonary vein is visualized where it connects to the left atrium (Fig. 10c). By again deflecting the silicone model slightly the location of the pulmonic semilunar valve can be visualized as a delineation between the right ventricle and the pulmonary artery (Fig. 10b). During the filling of the atria and ventricles with the liquid modeling compound, the right Atrioventricular (AV) valve (the tricuspid) was partially open and the valve location is delineated by a separation in the modeling material (Fig.10a), which explains why the right atrium and right ventricle remain partially attached by the cured silicone that passed through the open valvular orifice. 


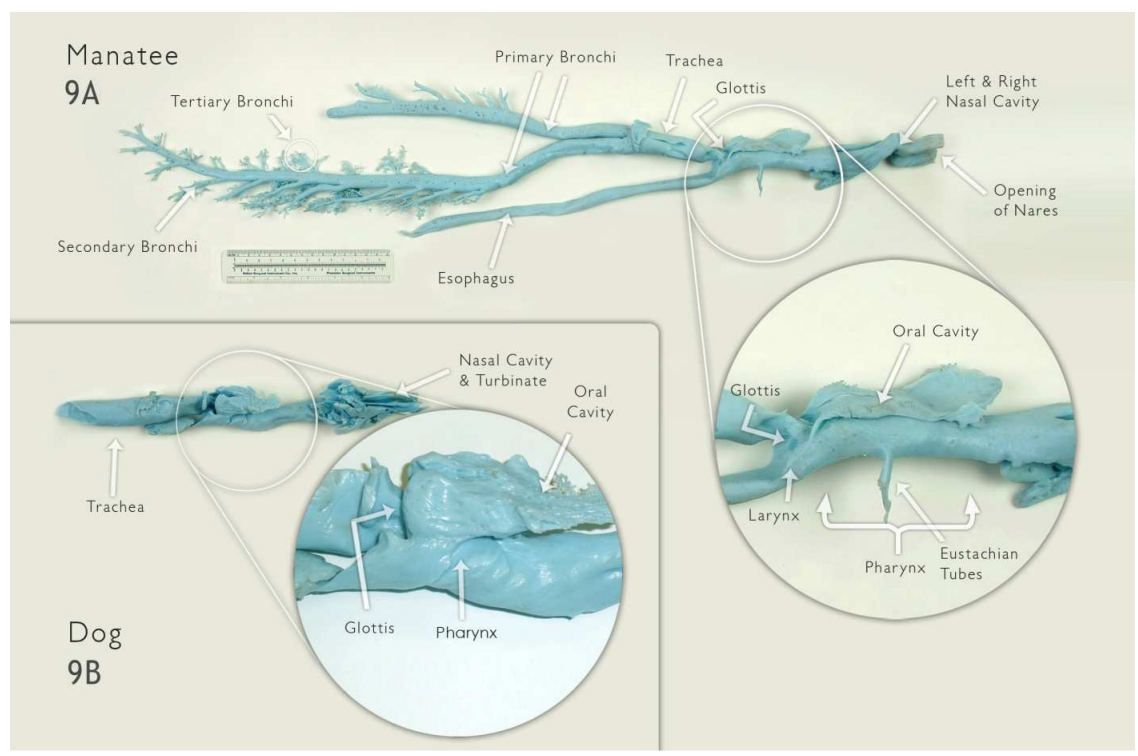

Fig. 9: Silicon model of the hollow spaces of the upper and lower respiratory tract in both the manatee and dog. (A) Silicon model of hollow spaces present in the manatee respiratory tract. To the left is the lower tract including the short trachea, primary bronchi, secondary and some tertiary bronchi. To the right is the upper tract including the pharynx, the left and right nasal cavity and part of the esophagus which branches off at the level of the pharynx. In the expanded view of Figure 9A the pharynx can be seen as well as one of the exceptionally large Eustachian tubes. (B) Silicon model of the hollow spaces of the dog respiratory tract. Note that the nasal cavity contains complex turbinate not present in the manatee nasal cavity. In both models the location of the glottis is indicated
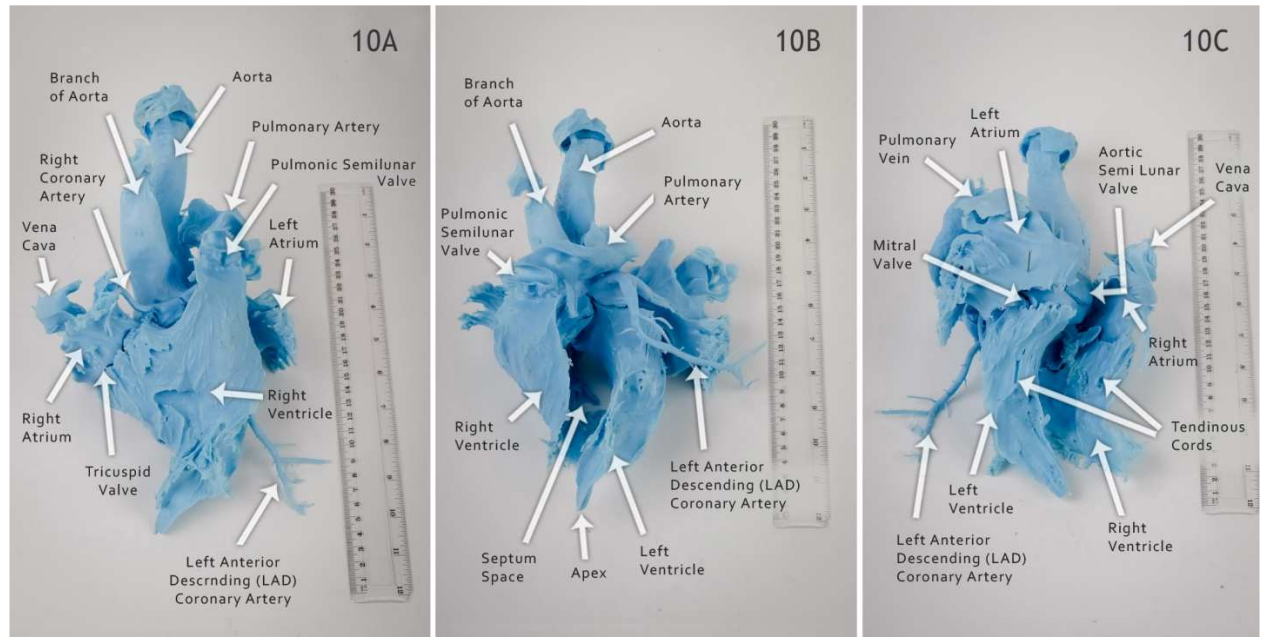

Fig. 10: Silicon model of the hollow spaces present in a bovine heart. (A) Lateral view: Right atria and ventricular spaces are modeled along with a portion of the right coronary artery and the left anterior descending (LAD) coronary artery. Vena cava, and location of tricuspid valve is seen as well as pulmonary artery and pulmonic semlunar valve, and aorta. (B) Ventral view: Portions of both the left and right ventricular spaces are modeled along with the separation between them produced by the septum. The apex can be visualized. LAD coronary artery is present. Pulmonary artery can be visualized and a branch of the aorta. (C) Dorsal view: Left atria and ventricular spaces are modeled. LAD is present. Grooves produced by the tendinous cords can be visualized as well as the pulmonary vein, location of the mitral valve and aortic semilunar valve 
However, the left AV valve (the mitral) was completely closed so no silicone could enter that valvular orifice. This accounts for the total separation between the left atrium and left ventricle (Fig. 10c) and for this reason we had to insert a dissecting pin through the left atrium and into the left ventricle to hold the model together (Fig. 10c). One can also visualize the impressions produced from the Cordae Tendineae in the silicon modeling material of the right (Fig. 10a) and left (Fig. 10c) ventricles.

\section{CONCLUSION}

We have developed an effective technique for modeling the internal respiratory structures of both the Florida Manatee (Trichechus manatus latirostris) and the dog. We suggest that this method can also be easily modified for use in other hollow organs as we demonstrated by modeling the interior spaces of a beef heart. The described silicone injection technique is both simple and inexpensive, requires no complex equipment and produces a detailed positive model, sufficiently flexible to allow relatively easy extraction, yet robust enough to withstand repeated handing and inspection. Using this technique in the manatee respiratory tract we were able to visualize the presence of unusually large Eustachian tubes in the pharyngeal region (Fig. 9a) which is supported by the earlier anatomical dissection studies performed by Rommel and Lowenstein (2001). Dogs do not possess such large Eustachian tubes (Fig. 9b and 9b expanded). We were also able to visualize both of the primary bronchi in the manatee model (Fig. 9a), along with secondary and even tertiary bronchi. These long, straight primary bronchi were expected because manatees possess unusual elongated dorsal lungs that run almost the entire length of the body, as well as hemi-diaphragms (Rommel and Reynolds, 2000).

\section{ACKNOWLEDGEMENT}

We would like to thank Katie Brill, Bron Bassett, Vince Bacalan, Kane Rigney, Garrett Chris Torno of the Marine Mammal Pathobiology Laboratory located in St Petersburg, Florida. We would like to thank Megan Whitt for her assistance in developing the silicon injection technique. We would also like to thank Nicole Adimey of the U.S. Fish Wildlife Service's Manatee Rescue, Rehabilitation Release Program. We are especially grateful to Gregory Rust and Jessica Murphy from Xavier University for their assistance in the preparation of the Figures for this publication. We would also like to express our appreciation to Janet $\mathrm{M}$.
Clem, DVM of the Cincinnati Animal Medical Center. This research was conducted under our U.S. Fish and Wildlife permit \#MA049136, was funded by Xavier University managed by the Midwest Florida Manatee Research Project, housed in the Biology Department at Xavier University in Cincinnati, Ohio.

\section{REFERENCES}

Hill, W.C.O., 1945. Notes on the dissection of two Dugongs. J. Mammal., 26: 153-175. http://www.jstor.org/stable/1375092

Murie, J., 1872. On the form structure of the Manatee (Manatus americanus). Trans. Zoological Soc. London, 8: 127-202. DOI: 10.1111/j.14697998.1872.tb08571.x

Rommel, S.J. and E. Reynolds, 2000. Diaphragm structure function in Florida Manatees (Trichechus manatus latirostris). Anat. Rec., 259: 41-51. PMID: 10760742

Rommel, S.A. and L.J. Lowenstein. 2001. Gross and Microscopic Anatomy of Marine Mammals. In: CRC Handbook of Marine Mammal Medicine, Dierauf, L.A. and F.M.D. Gulland (Eds.). CRC Press, Boca Raton, FL., pp: 129-163. ISBN: 08493-0839-9 